\title{
SHARPER Reaction Monitoring: Generation of a Narrow Linewidth NMR Singlet, without X-Pulses, in an Inhomogeneous Magnetic Field
}

\author{
Ariana B. Jones, Guy C. Lloyd-Jones, ${ }^{*}$ (i) and Dušan Uhrín*(i) \\ EaStCHEM School of Chemistry, University of Edinburgh, David Brewster Rd, Edinburgh EH9 3FJ, U.K.
}

Supporting Information

ABSTRACT: We report a new pure-shift method, termed SHARPER (Sensitive, Homogeneous, And Resolved PEaks in Real time) designed for the analysis of reactions and equilibria by NMR. By focusing on a single selected signal, SHARPER removes all heteronuclear couplings of a selected nucleus without the need to pulse on $\mathrm{X}$ channels, thus overcoming hardware limitations of conventional spectrometers. A more versatile decoupling scheme, termed sel-SHARPER, removes all heteronuclear and homonuclear couplings of the selected signal. Both methods are characterized by a periodic inversion of the active spin during the real-time acquisition. In addition to decoupling, they also compensate for pulse imperfections and magnetic field inhomogeneity, generating an extremely narrow singlet with a linewidth approaching limits dictated by the spin-spin relaxation. The decoupling and line narrowing effected by (sel)-SHARPER provide significant increases in the signal-to-noise (S/N) ratio. Increases of 20 -fold were routinely achieved for ${ }^{19} \mathrm{~F}$ detection. sel-SHARPER is also applicable to first- and higher-order ${ }^{1} \mathrm{H}$ spectra. The sensitivity gains are substantially greater for inhomogeneous magnetic fields, including dynamic inhomogeneity caused by gas sparging. The parameters of the pulse sequences have been analyzed in detail to provide guidelines for their most effective application. The considerable reduction in the detection threshold induced by (sel)-SHARPER make the technique particularly suited for in situ monitoring of reaction kinetics. The approach is illustrated by a ${ }^{19} \mathrm{~F}$ NMR study of the protodeboronation of an aryl boronic acid. Here, the high $\mathrm{S} / \mathrm{N}$ allowed reliable determination of the net protodeoboronation kinetics, and the excess line broadening of ${ }^{19} \mathrm{~F}$ singlets was utilized to characterize the boronic acid/boronate equilibrium kinetics. Oxidation of diphenylphosphine, monitored by ${ }^{31} \mathrm{P}$ NMR under optimized gas-flow conditions, demonstrated the high tolerance of SHARPER to dynamic inhomogeneity. The principles of the (sel)-SHARPER sequences are expected to find numerous applications in the design of new NMR experiments.

So olution-phase NMR is an indispensable tool for the in situ study of chemical reactions. However, it is not necessary to acquire a complete spectrum: the temporal concentration changes in reactants and/or products can be examined by following one signal, or a subset of resonances, simultaneously or sequentially, within the constraints presented by the reaction lifetime. Herein, we report a new NMR pulse sequence that we term SHARPER (Sensitive, Homogeneous, And Resolved PEaks in Real time; vide infra) designed for application within this framework. SHARPER belongs to a family of pure-shift NMR techniques ${ }^{1-5}$ to which it brings considerable benefits. Not only does it collapse multiplets to singlets, and substantially increases $\mathrm{S} / \mathrm{N}$, but it also addresses limitations of standard pure-shift approaches, including NMR hardware and magnetic field homogeneity requirements.

Pure-shift NMR methods have been the subject of considerable attention over the past few years due to their ability to significantly improve resolution and potentially also the sensitivity of detection of high natural abundance nuclei such as ${ }^{1} \mathrm{H}$ or ${ }^{19} \mathrm{~F}$. Particularly relevant to reaction monitoring are the real-time pure-shift methods ${ }^{6-9}$ that do not involve acquisition of multiple free induction decays (FIDs) associated with the pseudo-2D Zangger and Sterk approach. ${ }^{10}$ The realtime pure-shift methods manipulate spin systems during the directly detected acquisition period of NMR experiments by periodically inverting the passive spins while effectively leaving the spin states of the active spins unchanged. In practice, such spin manipulation is achieved by two consecutive spin inversion events, both of which are felt by the active, detected nuclei, whereas only one is registered by the passive, coupled spins. The overall $360^{\circ} / 180^{\circ}$ rotations experienced by these two spin groups are therefore equivalent to application of $0^{\circ} / 180^{\circ}$ pulses, a treatment that removes all intergroup couplings while allowing the chemical shift evolution of the active spins. The outcome is a pure-shift spectrum of either all $^{7}$ or a bandselected group of resonances. ${ }^{9}$ The former methods are general, but they often lead to a loss of signal. The band-selective

Received: June 23, 2017

Accepted: August 7, 2017

Published: August 7, 2017 
approach maintains all available magnetization and, by neglecting relaxation effects and pulse imperfections, increases the signal-to-noise $(\mathrm{S} / \mathrm{N})$ ratio relative to a reference spectrum, albeit only for a subset of signals. It is this aspect that is of particular interest to reaction monitoring, where maximizing sensitivity can be highly beneficial. Below, we present the design and implementation of a new pure-shift method, explore its scope and limitations, and demonstrate its application in the analysis of the kinetics of chemical reactions and equilibria by ${ }^{19} \mathrm{~F}$ and ${ }^{31} \mathrm{P}$ NMR.

\section{EXPERIMENTAL SECTION}

NMR Experiments. ${ }^{19} \mathrm{~F}$ spectra were acquired on a twochannel $400 \mathrm{MHz}$ Bruker Avance III NMR spectrometer equipped with a Prodigy probe or on a three-channel $400 \mathrm{MHz}$ Bruker Avance III NMR spectrometer equipped with a $5 \mathrm{~mm} \mathrm{z}$ gradient $\mathrm{BB}$ TBO ${ }^{1} \mathrm{H},{ }^{19} \mathrm{~F}$ probe. The ${ }^{19} \mathrm{~F}$ spectra of $\mathbf{1}$ in $\mathrm{CDCl}_{3}$ presented in Figure 2 were acquired using the following parameters: relaxation and nominal acquisition times of 1.5 and 17.4 s, respectively, with two dummy scans and four scans accumulated. The ${ }^{19} \mathrm{~F}$ SHARPER spectrum was acquired using the pulse sequence of Figure 1: 17 and $34 \mu \mathrm{s} 90^{\circ}$ and $180^{\circ}$ pulses, respectively, $n=256,34 \mathrm{~ms}$ chunk time, with an overall acquisition time per scan of $17.9 \mathrm{~s}$.

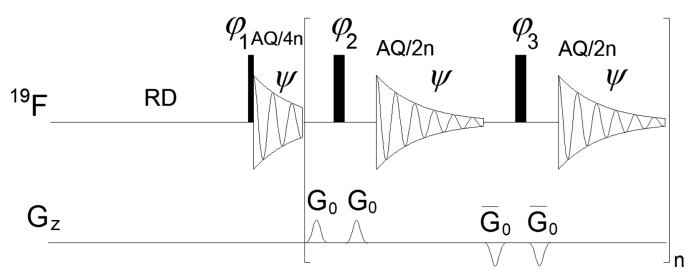

Figure 1. Pulse sequences of the SHARPER experiment. Narrow and wide filled rectangles represent $90^{\circ}$ and $180^{\circ}$ pulses, respectively. $\mathrm{RD}$ stands for relaxation delay. $300 \mu$ s sine-shaped PFGs were applied at $\mathrm{G}_{1}=1 \%$ followed by a $200 \mu$ s gradient recovery delay. The following phase cycle (minimum number of scans is 2 ) was used: $\varphi_{1}=2 x$, $2(-x), 2 y, 2(-y) ; \varphi_{2}=2(y,-y), 2(x,-x) ; \varphi_{3}=2(-y, y), 2(-x, x)$; and $\psi=2 x, 2(-x), 2 y, 2(-y)$.

The ${ }^{19} \mathrm{~F}$ SHARPER spectra of a mixture of $\mathbf{3}$ and $\mathbf{4}$ in a mixture of $1: 1 \mathrm{CD}_{3} \mathrm{OD} / \mathrm{H}_{2} \mathrm{O}$ presented in Figure 4 were acquired using the pulse sequence of Figure $3: n=128,13.6 \mathrm{~ms}$ acquisition chunks, $10 \mathrm{~ms} 180^{\circ}$ sinc pulse during the initial single PFG spin-echo (SPFGSE). Relaxation and nominal acquisition times of 10 and $3.49 \mathrm{~s}$ were used, respectively. Two dummy scans and four scans were accumulated. The spectra were processed by applying a $0.1 \mathrm{~Hz}$ exponential line broadening and a forward complex linear prediction (the stated signals half-height linewidths quote values without this additional line broadening). The ${ }^{19} \mathrm{~F}$ SHARPER spectra of Figure 5 were acquired using identical parameters as those used for the spectra of Figure 4, but with the $x, y, z, z^{2}, z^{3}, x y, x z$, and $y z$ shim corrections deviating by +500 units from their optimal values.

The ${ }^{19} \mathrm{~F}$ SHARPER spectra of 1 presented in Figure 6 were acquired using the pulse sequence of Figure 1. Relaxation and nominal acquisition times of 3 and $1.09 \mathrm{~s}$ were used, respectively. The loop parameter $n$ was set to $64,128,256$, or 512 , yielding acquisition chunks of $8.5,4.25,2.13$, and 1.06 $\mathrm{ms} ; 70 \mu \mathrm{s} 180^{\circ}{ }^{19} \mathrm{~F}$ pulses were applied during acquisition. Two dummy scans and two scans were accumulated. The spectra were processed by applying a $0.5 \mathrm{~Hz}$ exponential line broadening (the half-height linewidths are quoted without this additional line broadening). Gas sparging was applied as described in the text.

Spectra of $\mathrm{F}-3$ of 3 in $\mathrm{CD}_{3} \mathrm{OD} / \mathrm{H}_{2} \mathrm{O}$ (1:1 ratio) presented in Figure 7 were acquired using the SHARPER pulse sequence of Figure 1: relaxation delay of $3 \mathrm{~s}$, acquisition time of $0.89 \mathrm{~s}$, two dummy scans and two scans accumulated, $125 \mu \mathrm{s} 90^{\circ}$ rectangular excitation pulse and $250 \mu$ s rectangular $180^{\circ}$ pulses during the acquisition. The length of $180^{\circ}$ rectangular pulses was set to avoid perturbation of the F-1 and F-4 spins. The acquisition chunk lengths varied between 0.845 and $3.38 \mathrm{~ms}$. The spectra were processed using exponential line broadening of $0.5 \mathrm{~Hz}$ (the half-height linewidths are quoted without this additional line broadening). The $1 \mathrm{D}{ }^{19} \mathrm{~F}$ spectra were acquired using a $90^{\circ}$ excitation pulse and parameters used for the SHARPER spectra. Gas sparging was applied as described in the text.

$\mathrm{KOH}$ catalyzed protodeboronation of $2 / 2 \mathrm{a}$ in a $1: 1 \mathrm{H}_{2} \mathrm{O}$ / dioxane mixture was monitored by the acquisition of ${ }^{19} \mathrm{~F}$ NMR sel-SHARPER spectra (pulse sequence of Figure 3, $n=128$ ) in a stop-flow experiment (Figure 8). Two scans were acquired per spectrum using a relaxation delay of $1.5 \mathrm{~s}$ and an overall acquisition time of $4.99 \mathrm{~s}$ per scan, which consisted of $2.18 \mathrm{~s}$ of sampling (acquisition chunk time of $8.5 \mathrm{~ms}$ ) and $2.81 \mathrm{~s}(10 \mathrm{~ms}$ Gaussian pulses, $300 \mu$ s pulsed field gradients (PFGs), and 200 $\mu$ s gradient recovery delays). Two dummy scans were applied before the acquisition of the first spectrum only. Sixteen spectra were acquired with the first and the last starting 13 and $208 \mathrm{~s}$, respectively, after the mixing was triggered.

Spectra of 5/5a in a $1: 1 \mathrm{H}_{2} \mathrm{O}$ /dioxane mixture presented in Figure 9 were acquired using the sel-SHARPER pulse sequence of Figure 3: relaxation delay of $3 \mathrm{~s}$, acquisition time of $1.5 \mathrm{~s}$, two dummy scans and four scans accumulated, $93 \mu \mathrm{s} 90^{\circ}$ rectangular excitation pulse, $1 \mathrm{~ms} 180^{\circ}$ Gaussian pulse during the SPFGSE, and $186 \mu$ s rectangular $180^{\circ}$ pulses during the acquisition. The length of $180^{\circ}$ rectangular pulses was set to avoid perturbation of the other fluorine spins. The chunk length was set to $23.4 \mathrm{~ms}$. The $1 \mathrm{D}{ }^{19} \mathrm{~F}$ spectra were acquired using a relaxation delay of $3 \mathrm{~s}$, an acquisition time of $0.87 \mathrm{~s}$, two dummy scans, and eight scans. Samples were prepared by mixing $500 \mu \mathrm{L}$ of $0.1 \mathrm{M} 5$ containing $0.01 \mathrm{M}$ trifluoroacetic acid with increasing amounts of $0.1 \mathrm{M} 5$ in $0.1 \mathrm{M} \mathrm{KOH}$. In this way, the concentration of $\mathbf{5}$ was kept constant. The resulting concentrations of $\mathrm{KOH}$, together with obtained linewidths and chemical shifts, are reported in Table S4.

Line Shape Analysis. As indicated, the 5/5a exchange (eq $3)$ is fast on the chemical shift scales $\left(k_{\mathrm{a}} \gg \Delta \omega\right.$, where $\Delta \omega=$ $\Omega_{\mathrm{A}}-\Omega_{\mathrm{B}}$ and where $\Omega_{\mathrm{A}}$ and $\Omega_{\mathrm{B}}$ are the resonance frequencies of spins in sites A and B, respectively, in the absence of chemical exchange) and also on the relaxation time scale $(\Delta \omega$ $\gg \Delta R_{2}$ and $k_{\mathrm{ex}} \gg \Delta R_{2}$, where $\Delta R_{2}=\Delta R_{2 \mathrm{~B}}-\Delta R_{2 \mathrm{~A}}, R_{2 \mathrm{~A}}$ and $R_{2 \mathrm{~B}}$ are the relaxation rate constants for spins in sites $\mathrm{A}$ and $\mathrm{B}$, respectively, in the absence of chemical exchange, and $k_{\mathrm{ex}}=k_{\mathrm{a}}+$ $\left.k_{\mathrm{b}}\right)$. Under these circumstances, the relative site populations $p_{\mathrm{A}}$ and $p_{\mathrm{B}}\left(p_{\mathrm{A}}+p_{\mathrm{B}}=1\right)$ satisfy the balance relationship $p_{\mathrm{A}} k_{\mathrm{A}}=$ $p_{\mathrm{B}} p_{\mathrm{B}}$. If $p_{\mathrm{A}} \gg p_{\mathrm{B}}\left(\right.$ or $p_{\mathrm{B}} \gg p_{\mathrm{A}}$ ), the transverse relaxation rate constant $R_{2}$ of the population-averaged resonance line is given by eq 1 , where $R_{2}^{0}=p_{\mathrm{A}} R_{2 \mathrm{~A}}+p_{\mathrm{B}} R_{2 \mathrm{~B}}$. The position of the observed spectral line is given by eq 2

$$
\begin{aligned}
& R_{2}=R_{2}^{0}+p_{\mathrm{A}} p_{\mathrm{B}} \Delta \omega^{2} / k_{\mathrm{ex}} \\
& \Omega=p_{\mathrm{A}} \Omega_{\mathrm{A}}+p_{\mathrm{B}} \Omega_{\mathrm{B}}
\end{aligned}
$$


On the basis of the measured values of $\Omega_{\mathrm{A}}, \Omega_{\mathrm{B}}, \Omega, R_{2 \mathrm{~A}}$, and $R_{2 \mathrm{~B}}$, values of $p_{\mathrm{A}}$ and $k_{\mathrm{ex}}$ were calculated using eqs 1 and 2 .

\section{RESULTS AND DISCUSSION}

Alternative Pure-Shift Arrangements. In a pure-shift method designed for reaction monitoring, the r.f. carrier is placed on the chemical shift of a selected nucleus. This arrangement ultimately produces an exponential FID with zero chemical shift modulation. Under these circumstances, $180^{\circ}$ pulses can be repeatedly applied to the active spin. The effective $0^{\circ} / 180^{\circ}$ rotation of the detected/coupled protons, which is at the heart of broad-band decoupling of selected signals during indirect $^{11,12}$ or direct ${ }^{9,13}$ acquisition periods, can thus be replaced with a $180^{\circ} / 0^{\circ}$ element, maintaining all the attributes of a pure-shift methodology while realizing additional benefits.

In its basic form, the outlined pure-shift real-time experiment removes all heteronuclear couplings of an isolated nucleus, e.g., ${ }^{19} \mathrm{~F}$ in a fluorinated organic molecule, a motif common in contemporary medicinal chemistry. The experiment consists of an acquisition interrupted by a periodic application of nonselective $180^{\circ}$ pulses surrounded by low-level PFGs. As part of the repeating unit consisting of two such events, the two pairs of PFGs are of equal strength but opposite polarity, an arrangement that minimizes the disturbance of the static magnetic field and the lock circuitry (Figure 1).

By maintaining a $90^{\circ}$ phase difference between the initial $90^{\circ}$ excitation pulse and the subsequent $180^{\circ}$ pulses, this experiment possesses all of the attributes of the CPMG pulse sequence, ${ }^{14,15}$ compensating for static magnetic field inhomogeneity and pulse imperfections.

These properties have historically been used by Freeman and $\mathrm{Hill}^{16}$ for recording proton $J$-spectra. Such spectra showed extremely narrow linewidths, reflecting the true $T_{2}$ relaxation of protons. Freeman and Hill's experiment ${ }^{16}$ acquired a signal only at the peaks of individual spin-echoes, where the effects of magnetic field inhomogeneity are completely removed. In a different context, a spin-echo applied during an indirectly detected dimension of heterocorrelated experiments has been shown by Gochin et al. ${ }^{17}$ to remove signal broadening due to magnetic field inhomogeneity. Remarkably, as illustrated below, a significant line narrowing is maintained while relaxing the conditions applied for $J$-spectra to allow acquisition of signal in "chunks" of tens of milliseconds long, in a technique that we refer to as SHARPER.

Single Channel Heteronuclear Decoupling. The basic SHARPER method, outlined in Figure 1, removes all heteronuclear splittings, effectively performing broad-band heteronuclear decoupling without the need to pulse on $\mathrm{X}$ nuclei. This property can be very beneficial when studying fluorinated compounds, as the majority of NMR spectrometers, even the latest generation, are not equipped to deliver both ${ }^{1} \mathrm{H}$ and ${ }^{19} \mathrm{~F}$ r.f. pulses during the same experiment; in other words, recording ${ }^{19} \mathrm{~F}\left\{{ }^{1} \mathrm{H}\right\}{ }^{9}$ or ${ }^{1} \mathrm{H}\left\{{ }^{19} \mathrm{~F}\right\}$ spectra is rare. In addition, because SHARPER achieves decoupling by pulsing on the detected rather than decoupled nuclei, it simultaneously removes heteronuclear couplings of all $\mathrm{X}$ nuclei present. For example, when observing ${ }^{19} \mathrm{~F}$ in a partially deuterated compound, both ${ }^{1} \mathrm{H}$ - and ${ }^{2} \mathrm{H}$-involving couplings are removed, revealing the ${ }^{19} \mathrm{~F}\left\{{ }^{1} \mathrm{H},{ }^{2} \mathrm{H}\right\}$ signal for the nucleus of interest. Analogously, using SHARPER, ${ }^{1} \mathrm{H}\left\{{ }^{19} \mathrm{~F}\right\}$ signals can be recorded using highly sensitive inverse-detected triple-resonance cryoprobes, without the requirement to pulse on ${ }^{19} \mathrm{~F}$.
To illustrate the performance of the basic pulse sequence, a ${ }^{19} \mathrm{~F}$ SHARPER spectrum of fluorobenzene, $\mathbf{1}$, is presented in Figure 2. It is overlaid with a ${ }^{1} \mathrm{H}$-coupled ${ }^{19} \mathrm{~F}$ spectrum of $\mathbf{1}$

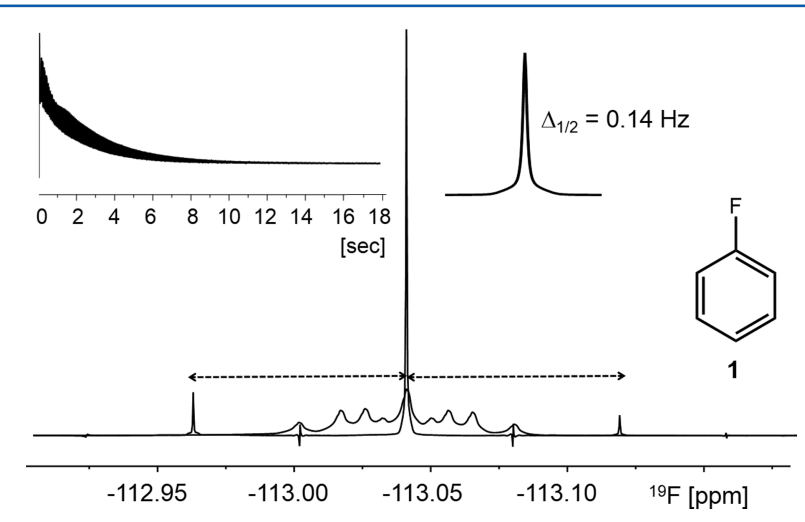

Figure 2. ${ }^{19} \mathrm{~F}$ SHARPER NMR spectrum $(400 \mathrm{MHz})$ of fluorobenzene, 1, acquired using the pulse sequence of Figure 1 and a $34 \mathrm{~ms}$ chunk time, overlaid with a ${ }^{1} \mathrm{H}$-coupled ${ }^{19} \mathrm{~F}$ spectrum of $\mathbf{1}$. The insets show an FID and the SHARPER signal obtained with no apodization. The position of the first side bands at 1000/34 $=29.41$ $\mathrm{Hz}$ is indicated. Full details are given in the Experimental Section.

containing a single ${ }^{19} \mathrm{~F}$ resonance as a triplet of triplets ( 9.1 and $5.8 \mathrm{~Hz}$ ) due to the splitting by pairs of chemically equivalent ortho and meta protons.

The ${ }^{19} \mathrm{~F}$ SHARPER spectrum of $\mathbf{1}$ contains an extremely narrow singlet (half-height linewidth, $\Delta_{1 / 2}=0.14 \mathrm{~Hz}$ ) that has 8 -fold the $\mathrm{S} / \mathrm{N}$ of the central line of the ${ }^{1} \mathrm{H}$-coupled ${ }^{19} \mathrm{~F}$ multiplet. In this example, a deliberately long chunk time of 34 ms was chosen to illustrate the robustness of the method. The odd-numbered chunking artifacts at $k /(2 *$ chunk time $)(k=1$, $3,5, \ldots)$ frequencies are largely suppressed by acquiring an initial half-chunk, while the side bands at $k /(1 *$ chunk time) decay rapidly with increasing $k$. A systematic exploration of the effects of the chunk length on the SHARPER spectra is presented later. The spectrum is clean with a flat baseline, devoid of the artifacts typical for standard ${ }^{19} \mathrm{~F}$ spectra caused by the presence of a fluorinated material in NMR probes. This feature is important for reliable signal integration in the analysis of reaction kinetics.

Hetero- and Homonuclear Decoupling. Minor modifications (Figure 3) of the basic pulse sequence make it more versatile and applicable to molecules with both hetero- and

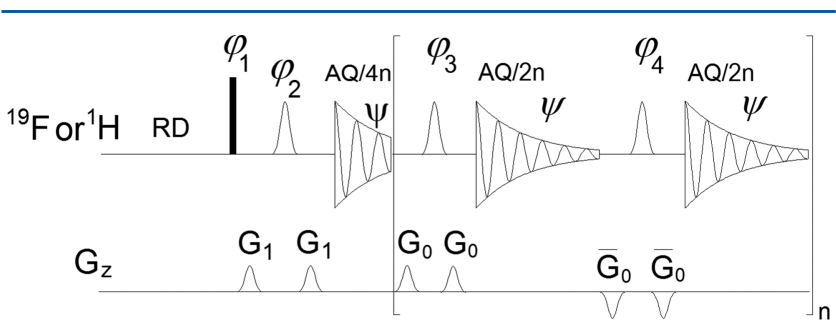

Figure 3. Pulse sequences of the sel-SHARPER experiment. The narrow filled rectangle represents a $90^{\circ}$ nonselective pulse, whereas open Gaussian shapes represent selective $180^{\circ}$ pulses. The description given in the caption to Figure 1 applies except for the phase cycling, which is as follows: $\varphi_{1}=4 x, 4(-x), 4 y, 4(-y) ; \varphi_{2}=2 y, 2 x, 2(-y)$, $4(-x), 2 y, 2 x, 2(-y) ; \varphi_{3}=4(y,-y), 4(x,-x) ; \varphi_{4}=4(-y, y), 4(-x, x)$; and $\psi=2 x, 4(-x), 2 x, 2 y, 4(-y), 2 y$ (minimum number of scans is two). $\mathrm{G}_{0}(1 \mathrm{~ms})$ was applied at $30 \%$. 
homonuclear couplings. The first modification selects the signal of interest by replacing the $90^{\circ}$ excitation pulse with a single PFG spin-echo ${ }^{18}$ containing a selective $180^{\circ}$ pulse surrounded by two PFGs. The second modification involves the use of selective inversion pulses during the acquisition. This can take the form of a carefully adjusted $180^{\circ}$ rectangular pulse, positioning signals of coupled spins between the lobes of its sinc inversion profile, or an arbitrary refocusing selective shaped pulse. Both approaches are collectively referred to as selective SHARPER or sel-SHARPER (Figure 3).

The benefits of sel-SHARPER are illustrated below through the $\mathrm{KOH}$ catalyzed protodeboronation of fluorinated aryl boronic acids. ${ }^{19,20}$ Protodeboronation of 2,3,6-trifluorophenyl boronic acid 2 , via boronate $2 \mathrm{a}$, in a 1:1 mixture of deuterated methanol and $\mathrm{H}_{2} \mathrm{O}$ yields 1,3,4-trifluorobenzene 3 and its isotopologue 2-[ $\left.{ }^{2} \mathrm{H}\right]-1,3,4$-trifluorobenzene 4 (Figure 4a). After

(c)

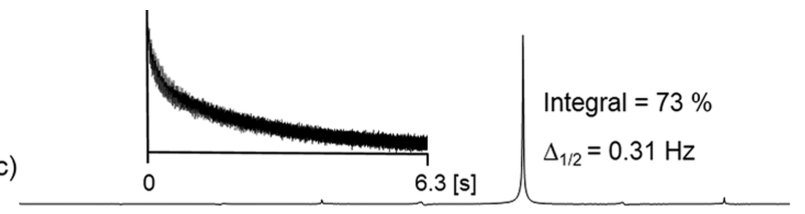

(b)

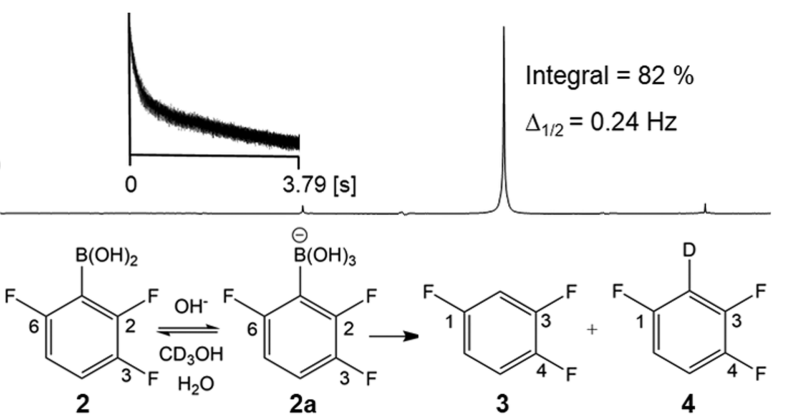

(a)

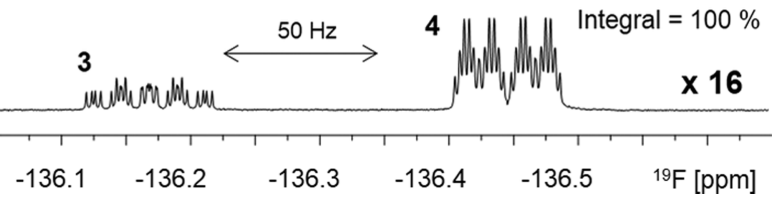

Figure 4. Partial ${ }^{19} \mathrm{~F}$ spectra focusing on F-1 resonances in a mixture of products 3 and 4 obtained by $\mathrm{KOH}$ catalyzed protodeboronation of 2 in $\mathrm{CD}_{3} \mathrm{OD} / \mathrm{H}_{2} \mathrm{O}$. (a) ${ }^{1} \mathrm{H}$-coupled ${ }^{19} \mathrm{~F}$ spectrum (scaled up 16 times) (b) and (c) sel-SHARPER spectra of 4 acquired using the pulse sequence of Figure 3: $205 \mu \mathrm{s} 180^{\circ}$ rectangular (b) or $10 \mathrm{~ms}$ Gaussian pulses (c) during the acquisition. The insets shows FIDs; $\Delta_{1 / 2}$ and integral intensities relative to that of F-1 in (a) are given. Full details are given in the Experimental Section.

complete consumption of 2 , the ${ }^{19} \mathrm{~F}$ spectrum of the resulting mixture of 3 and 4 shows six ${ }^{19} \mathrm{~F}$ signals, split by numerous ${ }^{19} \mathrm{~F}-{ }^{19} \mathrm{~F},{ }^{1} \mathrm{H}-{ }^{19} \mathrm{~F}$, and ${ }^{2} \mathrm{H}-{ }^{19} \mathrm{~F}$ couplings (4 only). These complex ${ }^{19} \mathrm{~F}$ multiplets are up to $50 \mathrm{~Hz}$ wide, with ${ }^{19} \mathrm{~F}$ resonances from 3 and 4 separated by ${ }^{1} \mathrm{H} /{ }^{2} \mathrm{H}$ isotope shifts (Table $S 1$ and Figure $S 1$ ). Focusing on the F-1 signal of 4 , a ${ }^{1} \mathrm{H},{ }^{2} \mathrm{H}$-coupled ${ }^{19} \mathrm{~F}$ spectrum (Figure $4 \mathrm{a}$ ) and two ${ }^{19} \mathrm{~F}$ selSHARPER spectra (Figure $4 b, c$ ) are compared. The two selSHARPER spectra were acquired using a $10 \mathrm{~ms}$ sinc pulse during the initial SPFGSE selecting the signal of F-1, whereas $205 \mu \mathrm{s} 180^{\circ}$ rectangular (Figure $4 \mathrm{~b}$ ) or $10 \mathrm{~ms}$ Gaussian pulses ${ }^{21}$ (Figure 4c) were applied during the acquisition, respectively. The length of the rectangular pulses was adjusted to position the F-3 and F-4 resonances of 4 between the lobes of their sinc inversion profile, to prevent the spin states of these nuclei from changing. In both instances, the collapse of the F-1 multiplet in the sel-SHARPER spectra delivers significant sensitivity gains: the $\mathrm{S} / \mathrm{N}$ is 20 -fold greater than that in the ${ }^{1} \mathrm{H},{ }^{2} \mathrm{H}$-coupled ${ }^{19} \mathrm{~F}$ spectrum. The signal loss due to real-time decoupling is small, with 82 and $73 \%$, respectively, of the integral intensities preserved in the two sel-SHARPER spectra relative to the reference 1D spectrum. The insets in Figures 4, panels b and c, show FIDs, where a more complete decay is seen when Gaussian pulses were applied. This is mainly because the actual acquisition time has almost doubled from the nominal value of 3.49 to $6.3 \mathrm{~s}$, in this instance, whereas only a modest increase to $3.79 \mathrm{~s}$ accompanied the use of rectangular pulses. In both cases, the signals have not yet decayed to zero intensity. The small increase in the linewidths, from $\Delta_{1 / 2}$ of 0.24 to $0.31 \mathrm{~Hz}$, is thus caused by the additional relaxation during the longer Gaussian pulses.

Line-Narrowing Properties of SHARPER. It can sometimes be difficult to achieve or maintain perfect magnetic field homogeneity when analyzing reactions in situ by NMR. Examples include monitoring slow reactions in nondeuterated solvents without autoshim, reactions taking place in multiphase environments, or reactions that require or induce agitation, e.g., for gas-liquid phase transfer or gas generation as a reaction evolves. To test the ability of the SHARPER to improve analysis of reactions conducted in inhomogeneous magnetic fields, the shim corrections were deliberately miss-set and the sample of $\mathbf{3}+\mathbf{4}$ was reacquired using otherwise identical parameters. It can be see (Figure 5) that sel-SHARPER very

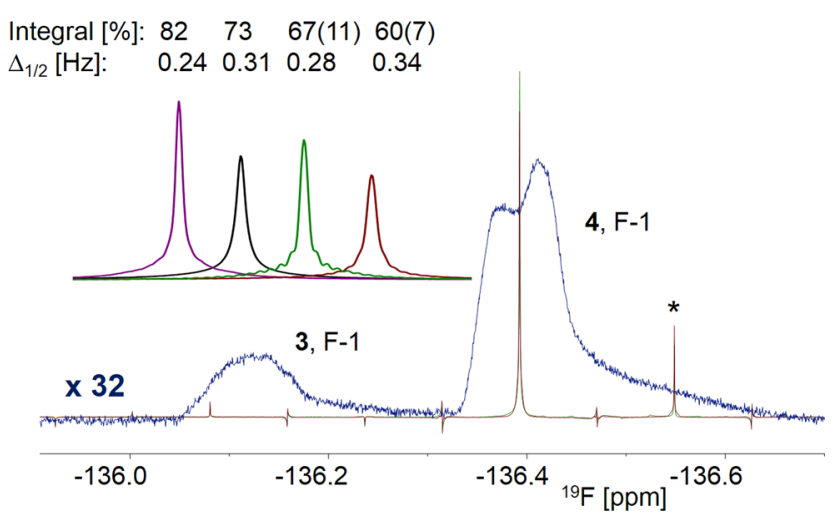

Figure 5. Overlay of ${ }^{19} \mathrm{~F}$ spectra as presented in Figure 4 but acquired in an inhomogeneous magnetic field with $x, y, z, z^{2}, z^{3}, x y, x z$, and $y z$ shim corrections offset by +500 units from their optimal values. The ${ }^{1} \mathrm{H}$-coupled ${ }^{19} \mathrm{~F}$ spectrum (blue, scaled $\times 32$ ) and sel-SHARPER spectra used rectangular (green) and Gaussian (brown) inversion pulses. The inset shows, left to right, the F-1 signals from spectra of Figure $4 \mathrm{~b}$ (violet) and $4 \mathrm{c}$ (black) and the two corresponding signals acquired under miss-shimmed conditions. Signal attributes $\left(\Delta_{1 / 2}\right.$ and integral intensities) are given relative to those presented in Figure 4. The values in parentheses belong to the side band at $-136.55 \mathrm{ppm}$ labeled with an asterisk. Full details are given in the Experimental Section.

efficiently compensates for the magnetic field inhomogeneity, resulting only in a minor deterioration of the quality of the spectra compared to samples run with ideal shims (Figure 4). The intensities of the main signal of F-1 of 4 dropped only by $18 \%$ with an increase in linewidth of less than $0.04 \mathrm{~Hz}$.

The missing signal intensity in the SHARPER singlet of $\mathbf{4}$ was directed toward the first side band upnfield of the main 
signal, where a fraction of the spins resonate; the downfield symmetrical side band is absent. This observation suggests that a decreased chunk time will better compensate for large magnetic field inhomogeneity as this positions the side bands further away from the main resonance, outside of the signal broadened by the magnetic field inhomogeneity. The efficacy of this approach is illustrated in Figure S2. Careful inspection of the spectra presented in Figure S2 shows that the sum of the integrals of the main peak and the side bands remains practically constant. Hence, inclusion of side bands during quantitation is advisible if a reduced chunk time is not desired (see discussion below).

A broad-band method for acquisition of pure-shift NMR spectra, UPSIF (ultra-high-resolution pure shifts in inhomogeneous fields), was recently reported. ${ }^{22}$ It generates highresolution $1 \mathrm{D}$ and $2 \mathrm{D}$ spectra by sampling the intermolecular zero quantum coherences between the solute and solvents during a constant-time indirect dimension, producing impressive signal narrowing. This, however, comes at the cost of sensitivity (up to 90\%) and time (tens of FIDs are required to reconstruct a single time point). The UPSIF method therefore is suited to monitoring slower reactions and those where the amount of material is not a limiting factor. It is also better suited for applications where severe spectral overlap exists.

Gas Sparging. As an example of the ability of SHARPER to compensate for "dynamic" magnetic field inhomogeneity, spectra of fluorobenzene $\mathbf{1}$ were recorded while nitrogen was bubbled through the solution (Figure 6). Shorter chunk times

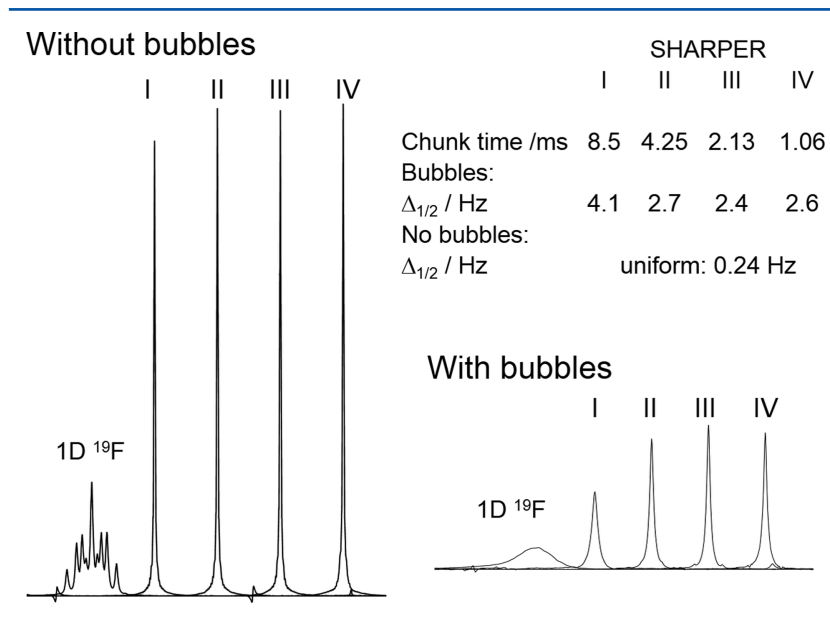

Figure 6. ${ }^{19} \mathrm{~F}$ SHARPER spectra without (left) and with $\mathrm{N}_{2}$ sparging (right) plotted on an identical vertical scale. ${ }^{1} \mathrm{H}$-coupled ${ }^{19} \mathrm{~F} 1 \mathrm{D}$ spectra of 1 obtained under these conditions are also shown. The signal half-widths are stated as a function of the chunk time. Full details are given in Experimental Section.

(i.e., shorter spin-echoes) are better able to restore the line shape than longer ones: fluctuation of the magnetic field inhomogeneity at time scales faster than the spin-echo times cannot be compensated for. Even though the SHARPER singlets during gas sparging are 10 times wider than those in regular samples, this still represents an $\sim 8$-fold increase in $\mathrm{S} / \mathrm{N}$ compared to the $1 \mathrm{D}{ }^{19} \mathrm{~F}$ spectrum acquired under the same conditions.

By using lower bubbling rates, we were able to obtain narrower linewidths, but the signal intensities varied. When subsequently optimizing the parameters with a view to following the reaction kinetics, $\mathrm{N}_{2}$ gas bubbling was found to impact the stability of the signal integrals, although this occurred more so at relatively low bubbling rates. When the $\mathrm{N}_{2}$ flow rate was maintained at about $180 \mathrm{~mL} / \mathrm{h}$ to generate a dynamic bubble population of $\sim 1 / \mathrm{cm}(z$-axis), the integrals became substantially more stable (Figure S3).

The rationale behind this observation is that at lower $\mathrm{N}_{2}$ flow rates there will be instances where one or no bubble is present in the active volume of the coil (approx $1.5 \mathrm{~cm}$ in length), whereas at a higher $\mathrm{N}_{2}$ flow rate, two bubbles will always occur. Even though displacement of solution from the active volume by the gas bubbles reduces the number of spins contributing to the measured signal, the integrals are more reliable. To demonstrate these effects, SHARPER spectra were acquired focusing on the F-3 signal of 3 . An overlay of $32 \mathrm{ID}$ and SHARPER spectra acquired at two bubbling rates (fast and slow) are presented in Figure 7.

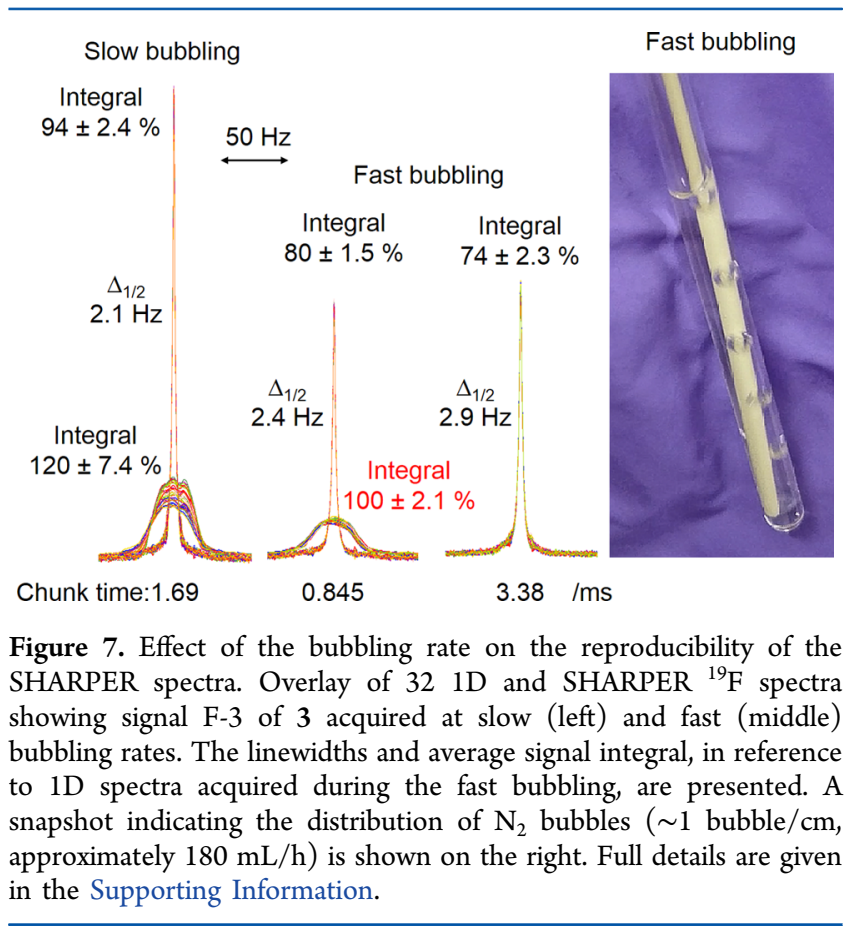

Inspection of the spectra showed that (i) benefits of SHARPER are largely preserved under significant gas flow (the linewidths only increase from $0.5 \mathrm{~Hz}$ to $<2.5 \mathrm{~Hz}$ ), (ii) the signal integrals of SHARPER spectra are reduced in comparison to $1 \mathrm{D}$ spectra integrals, however, their scatter is smaller, (iii) as discussed above, better reproducibility is achieved for gas flows that generate $\sim 1$ bubble $/ \mathrm{cm}$ (fast) compared to a $\sim 0.5$ bubble $/ \mathrm{cm}$ (slow) regime (this is true for both $1 \mathrm{D}$ and SHARPER spectra), and (iv) lowering the chunk time reduces the linewidths and scatter of the signal integrals in the SHARPER spectra.

It should be noted that if one signal needs to be selected during the initial SPFGSE of the sel-SHARPER sequence (Figure 3), the duration of the selective pulse and the strength and duration of PFGs should be kept to a minimum; otherwise, the SPFGSE becomes a source of significant signal losses and intensity variations due to intense convection.

As seen in the above examples, the interplay between the acquisition chunk time and the experimental conditions are important for obtaining high-quality SHARPER spectra. To assist users in achieving optimal performance, the effects of the 
chunk length on the overall performance of the SHARPER experiment were systematically explored. In addition, the effects of frequency mismatch and pulse misscalibration were also investigated. The findings are detailed in the Supporting Information, with only the main conclusions summarized herein.

Factors Affecting SHARPER Spectra. The most important factors are found to be the chunk length and the choice of method for selective inversion of the active spin (for details, see Figures S4 and S5 and Tables S2 and S3). The optimal setting depends on the nature of the investigated molecule, its relaxation properties, and the complexity of its spectrum. While short chunk times are generally beneficial, for couplings of $<15$ $\mathrm{Hz}$, chunk lengths of tens of milliseconds are perfectly acceptable and are even preferable when long selective inversion pulses are required for spin inversion. These long pulses increase the effective spin-spin relaxation, broadening the spectral lines. This is common to all band-selective realtime pure shift methods; ${ }^{9,13}$ however, only SHARPER compensates for magnetic field inhomogeneity through the CPMG scheme. The band-selective methods effectively apply a $0^{\circ}$ pulse to the active nuclei between acquisition chunks and hence cannot act in a similar manner. SHARPER signals will therefore always be narrower under comparable circumstances. To maximize these benefits, it is preferable to use carefully adjusted short rectangular $180^{\circ}$ pulses to avoid inversion of spins coupled to the observed spin. Noncoupled spins can be inverted without affecting the outcome as they will have already been eliminated by the initial SPFGSE that can be made highly selective. These considerations are particularly important for crowded ${ }^{1} \mathrm{H}$ spectra. As discussed above, when gas is bubbled through the sample, short chunk times and short inversion pulse perform better. It should be noted that the effect of magnetic field inhomogeneity is reduced in spatially selective pure-shift experiments. ${ }^{23}$ These have been applied in reaction monitoring; however, their sensitivity is much reduced due to spatial selection.

In the context of reaction monitoring, signals can change their resonance frequency; e.g., when the $\mathrm{pH}$ of the medium changes with conversion, phase separation takes place, or the magnetic field drifts when experiments are performed on unlocked samples over a long period of time. As SHARPER requires the r.f. pulses to be applied on resonance, substantial drift of signals will have negative consequences. We have demonstrated (see Figure S6) that SHARPER can be used for quantitative analysis of reaction kinetics even when signals are moving during monitoring, but it is essential to include side bands in the data analysis. Short chunk times reduce the leakage of the signal into side bands and are a necessity when larger movements of resonances are anticipated. Similarly, as discussed above, inclusion of side bands for quantitative evaluation is required when severe magnetic field inhomogeneity is present. Alternatively, reduced chunk times may push the side bands outside of the area of signal intensity. Finally, the effect of pulse accuracy (or excessive B1 inhomogeneity) on the quality of the SHARPER sequence was investigated (Figure S7). The results demonstrate remarkable tolerance of SHARPER to pulse imperfections; nevertheless, pulse calibration to an accuracy of $\pm 10 \%$ is recommended to maximize the $\mathrm{S} / \mathrm{N}$ and the line shape quality.

Although SHARPER was applied above to ${ }^{19} \mathrm{~F}$, there is no fundamental difference when acquiring ${ }^{1} \mathrm{H}$ SHARPER spectra, as illustrated on an example of vincamine (Figure S8). This is particularly true for first-order multiplets and X parts of the higher-order spin systems, such as the ABCX spin system consisting of four aromatic vincamine protons (Figure S8b, panel A). When applied to the A part of an ABMRX spin system of the same molecule, a $80 \mathrm{~ms}$ sinc pulse had to be used in order not to invert proton B. This lead to a nonuniform inversion of individual lines of this multiplet, which in combination with fast relaxation of the $\mathrm{CH}_{2}$ protons decreased the signal intensity; nevertheless, a singlet was produced (Figure S8c, panel B).

Reaction Monitoring and Chemical Exchange. As an example of reaction monitoring using SHARPER, a time-course for protodeboronation ${ }^{19,20}$ of $\mathbf{2}$ (the reaction scheme is shown in the inset of Figure 4a) was recorded. The reaction was initiated by a stop-flow system to effect fast and efficient mixing of a solution of boronic acid 2 with a solution of $\mathrm{KOH}$, in dioxane/water. Separate sel-SHARPER experiments focused on each of the ${ }^{19} \mathrm{~F}$ signals arising from reactant 2 and product 3 (see Figure S9). In the runs shown in Figure 8, the magnetic

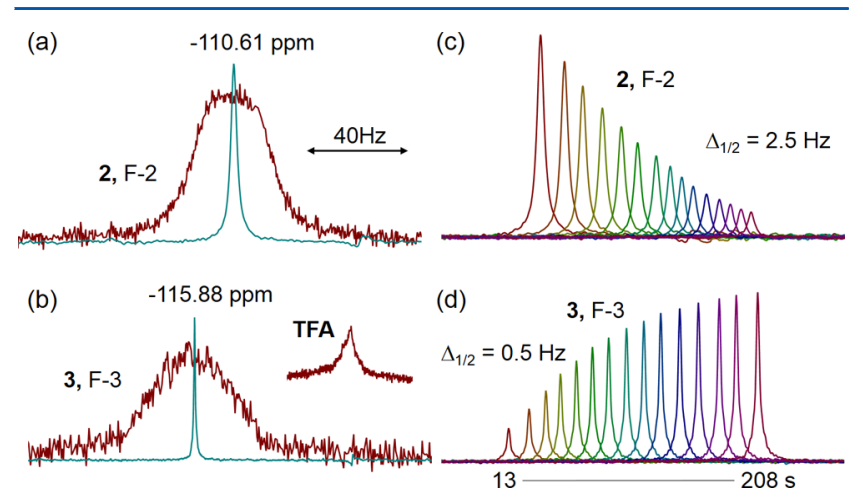

Figure 8. Monitoring protodeboronation of 2. (a, b) Overlay of ${ }^{19} \mathrm{~F}$ signals of reactant 2 and product 3 from a ${ }^{1} \mathrm{H}$-coupled ${ }^{19} \mathrm{~F}$ spectrum of the reaction mixture and sel-SHARPER spectra of F-2 and F3. The inset in (b) shows the signal of trifluoracetic acid, indicating poor magnetic field homogeneity. (c, d) Intensity changes over $3.5 \mathrm{~min}$. The $\Delta_{1 / 2}$ values are indicated. Full details are given in the Experimental Section.

field was deliberately made nonhomogeneous, as evidenced by the imperfect line shape of the trifluoroacetic acid (see inset in Figure $8 \mathrm{~b}$ ) used as a $\mathrm{pH}$ stabilizer in the stock solution of 2 . The signals arising from F-2 in 2, which convert to F-3 in 3 via protodeboronation $\left(k_{\mathrm{obs}}=1.28 \times 10^{-2} \mathrm{~s}^{-1}\right)$, are intense and narrow. This stands in contrast to the very broad multiplets observed in standard $1 \mathrm{D}{ }^{19} \mathrm{~F}$ NMR spectra. The numerous scalar couplings and line broadening by the magnetic field inhomogeneity make extraction of the kinetic data significantly less reliable than from sel-SHARPER spectra.

All of the ${ }^{19} \mathrm{~F}$ signals in the reaction product $(1,3,4$ trifluorobenzene 3) were sharp $\left(\Delta_{1 / 2}=0.5 \mathrm{~Hz}\right)$. However, the ${ }^{19} \mathrm{~F}$ signals of reactant $\mathbf{2}$ were notably broader, with linewidths that ranged from 1.6 to $3.5 \mathrm{~Hz}$, depending on the position of the ${ }^{19} \mathrm{~F}$ nuclei relative to the boron center (C1). The (sel)-SHARPER sequence does not compensate for relaxation caused by fast chemical exchange. Hence, excess line broadening (i.e., $\Delta_{1 / 2}>0.5 \mathrm{~Hz}$ ) can be interpreted in terms of dynamics associated with underlying equilibrium processes. In the case of 2 , this involves rapid, $\mathrm{pH}$-dependent interconversion of $\mathbf{2}$ with a 2,3,6-trifluorophenyl trihydroxy boronate anion, $\mathbf{2 a}$ (see inset to Figure 4a), a key intermediate in the protodeboronation process. ${ }^{19}$ The extent of excess line 
broadening depends on (i) the rate of equilibration, (ii) the chemical shift differences between the two exchanging ${ }^{19} \mathrm{~F}$ sites, and (iii) the equilibrium population of 2 versus $2 \mathbf{a}$, as dictated by $\mathrm{pH}$, or $[\mathrm{OH}]^{-}$.

Analogous excess line broadening is observed with 2,4difluorophenyl boronic acid, 5 (Figure 9), which undergoes

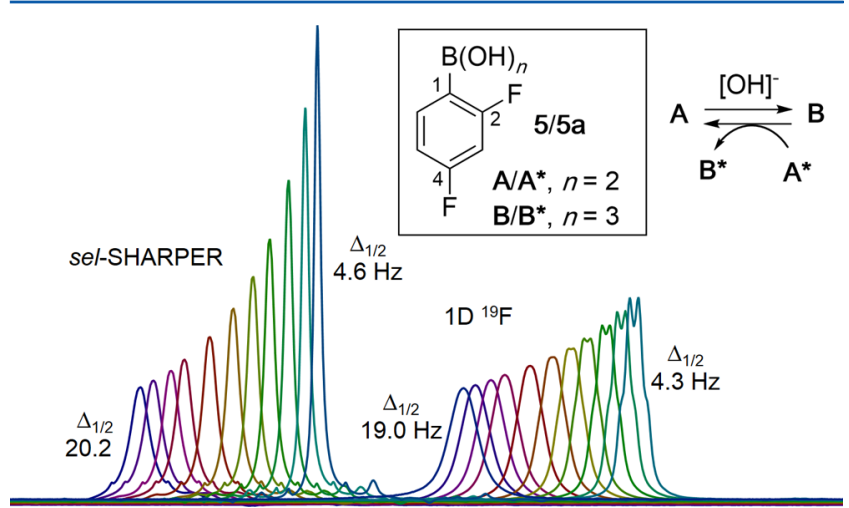

Figure 9. Overlay of ${ }^{19} \mathrm{~F}$ sel-SHARPER (left) and ${ }^{19} \mathrm{~F}$ 1D (right) signals of F-2 in $\mathbf{5 / 5 a}$ undergoing the equilibrium shown in the inset. The $\Delta_{1 / 2}$ values, determined as explained in the text, are indicated for the first and last titration points using increasing $\mathrm{KOH}$ concentration. For further details, see the Experimental Section.

much slower protodeboronation than $2\left(k_{\text {rel }} \leq 10^{-5}\right)$. This feature allowed a much more detailed analysis of the dynamics between reactant 5 and reaction intermediate $5 \mathbf{a}$. As with $\mathbf{2 / 2 a}$, only one signal is observed for each fluorine nucleus due to the fast exchange between $\mathbf{5}$ and $\mathbf{5 a}$ on the NMR time scale. When superstoichiometric $\mathrm{OH}^{-}$is added, the signals sharpen as the proportion of 5a approaches $100 \%$ (see the F-2 signals in Figure 9).

Ishihara $^{24}$ has studied the boric acid (A)-borate (B) equilibrium (eq 3 ) in detail by ${ }^{11} \mathrm{~B}$ NMR. It was shown that $k_{\mathrm{a}}$ increases linearly with $\left[\mathrm{OH}^{-}\right]$, approaching a constant value: $k_{\mathrm{a}}=k_{2} c_{\mathrm{T}}$, when $\left[\mathrm{OH}^{-}\right] \gg K_{\mathrm{b}}$ (where $c_{\mathrm{T}}=c_{\mathrm{A}}+c_{\mathrm{B}}$, the total boron concentration; and $K_{\mathrm{b}}$ is the basicity constant of the borate, $\mathrm{B}$ ). Thus, NMR analysis of the apparent exchange rate (eq 3) at known $c_{\mathrm{T}}$ allows determination of $k_{2}\left(\mathrm{M}^{-1} \mathrm{~s}^{-1}\right)$, the intermolecular rate constant for degenerate $\mathrm{OH}^{-}$exchange between pairs of boric and borate reactants (eq 4).

$$
\begin{aligned}
& \mathrm{A} \underset{k_{\mathrm{b}}}{\stackrel{k_{\mathrm{a}}}{\rightleftharpoons}} \mathrm{B} \\
& \mathrm{A}^{*}+\mathrm{B} \stackrel{k_{2}}{\rightleftharpoons} \mathrm{B}^{*}+\mathrm{A}
\end{aligned}
$$

When applied to our system, $\mathrm{A}, \mathrm{A}^{*}$ and $\mathrm{B}, \mathrm{B}^{*}$ are $\mathbf{5}$ and $\mathbf{5 a}$, respectively. To determine $k_{2}$, we analyzed ${ }^{19} \mathrm{~F}$ sel-SHARPER and $1 \mathrm{D}{ }^{19} \mathrm{~F}$ spectra of a series of $\mathbf{5} / \mathbf{5 a}$ samples $\left(c_{\mathrm{T}}=0.1 \mathrm{M}\right)$ containing 0.1-1.7 equiv of exogenous $\mathrm{OH}^{-}$. The excess line broadening was determined from the measured linewidths of the sel-SHARPER spectra or by matching the signals in 1D spectra with those generated by applying an appropriate line broadening to the spectrum of $\mathbf{5 a}$ measured at larger excess of $\mathrm{OH}^{-}$. Comparison of the methods revealed that the $\Delta_{1 / 2}$ values obtained by SHARPER are systematically larger, by factors of 1.069 for F-2 and 1.062 for F-4. This increase is due to additional relaxation occurring during the PFGs and $180^{\circ}$ pulses applied between the acquisition chunks of the SHARPER acquisition period. When $t_{\text {chunk }} \gg \delta_{\text {(PFG+pulses) }}$, a linear approximation can be used to calculate a linewidth correction factor (eq 5; Figure S10).

$$
t_{\text {chunk }} /\left(t_{\text {chunk }}+\delta_{(\mathrm{PFG}+\text { pulses })}\right)
$$

For our experimental setting, this factor is $1 / 1.06$, which is in a very good agreement with the average determined values of $1 /$ 1.066. After correction of the $\Delta_{1 / 2}$ values, eqs 1 and $2,{ }^{25}$ given in the Experimental Section, were used to analyze the data (Table S4). For F-2, the population of A (i.e., 5), $p_{\mathrm{A}}$, varied between 0.022 and 0.167 , yielding $k_{\mathrm{ex}}=132000 \pm 13500$ and $134000 \pm 16000 \mathrm{~s}^{-1}$, as an average of 11 measurements, for SHARPER and $1 \mathrm{D}{ }^{19} \mathrm{~F}$ spectra, respectively. For F-4, $p_{\mathrm{A}}$ varied between 0.018 and 0.150 , yielding $k_{\mathrm{ex}}=146000 \pm 6800$ and $149000 \pm 8200 \mathrm{~s}^{-1}$ for SHARPER and 1D ${ }^{19} \mathrm{~F}$ spectra, respectively (Figure S11). As eqs 1 and 2 are valid only when there is very large excess of either A or B (i.e., $\mathbf{5}$ or $\mathbf{5 a}$ ), we simulated a two-site exchange process according to the expressions derived by Rogers and Woodbrey ${ }^{26}$ (Figure S12). Using experimental values of $\Delta \omega$ for F-2 $(1061 \mathrm{~Hz})$ and F-4 $(3552 \mathrm{~Hz})$, with $p_{\mathrm{A}}$ ranging from 0.001 to 0.16 and $k_{\text {ex }}$ between 110000 and $145000 \mathrm{~s}^{-1}$, allowed determination of $\Omega$ and $R_{2}$. These were then used to calculate $k_{\mathrm{ex}}$, according to eqs 1 and 2 . The values for $k_{\text {ex }}$ obtained in this way showed relative standard deviations between 1 and 3\% (data not shown) across the stated $p_{\mathrm{A}}$ range, indicating that the use of eqs 1 and 2 is justified.

The observed differences between the F- 2 and F- 4 based $k_{\text {ex }}$ values therefore are not a consequence of the choice of data interpretation formalism. They arise because the observed positions of the spectra lines are affected not only by the exchange but also by additional factors such as changing $\mathrm{pH}$ (see the Supporting Information for a full discussion). Despite the observation that the $k_{\mathrm{ex}}$ values differ slightly depending on which of the two sites (F-2 versus F-4) is analyzed, the rate determined for degenerate intermolecular $\mathrm{OH}$ transfer between 5 and $5 \mathbf{a}\left(k_{2}=1.3 \pm 0.2 \times 10^{6} \mathrm{M}^{-1} \mathrm{~s}^{-1}\right)$ compares well with those reported by Ishihara for boric acid/borate $\left(k_{2}=2.6 \times 10^{6}\right.$ $\left.\mathrm{M}^{-1} \mathrm{~s}^{-1}\right)^{24}$ and for methylboronic acid/methylboronate $\left(k_{2}=\right.$ $\left.0.70 \times 10^{6} \mathrm{M}^{-1} \mathrm{~s}^{-1}\right) .^{27}$

Oxidation of Diphenylphosphine. To demonstrate the performance of SHARPER in reaction monitoring under gasflow conditions, the aerobic oxidation of diphenylphosphine, 6 , to diphenylphosphine oxide, 7, was followed in toluene- $d_{8}$. This example also demonstrates the ability of SHARPER to decouple large heteronuclear coupling constants $\left({ }^{1} J_{\mathrm{PH}}\right.$ of $215.9 \mathrm{~Hz}$ in this case). Such large coupling required a reduction in the chunk time to around $1 \mathrm{~ms}$ to maximize the benefits of SHARPER (see Figure S13). Short chunk times are also required to minimize the effects of gas bubbles. Figure 10 shows integral intensities of the ${ }^{31} \mathrm{P}$ signal of 6 during the oxidation, including examples of spectra. The original data are presented in Figure S14. It can be seen that the SHARPER signals show smaller immediate fluctuation of integral intensities, narrow linewidths, and a significantly larger $\mathrm{S} / \mathrm{N}$ ratio (despite the overall drop of integral intensities of $\sim 40 \%$ relative to the $1 \mathrm{D}$ spectra). These attributes of the SHARPER pulse sequence make it a useful tool for monitoring chemical reactions under challenging conditions of gas sparging.

\section{CONCLUSIONS}

A simple and robust pure-shift NMR method, termed SHARPER, has been developed, and its parameters were 


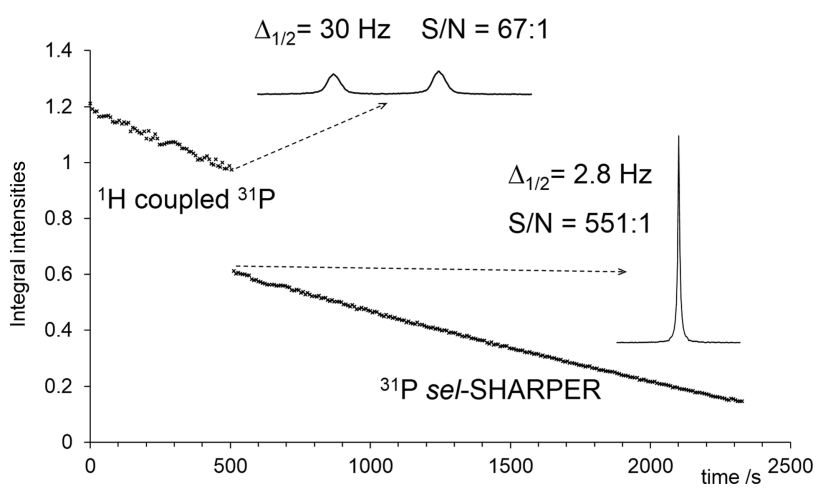

Figure 10. Integral intensities of the ${ }^{31} \mathrm{P}$ signal of 6 during aerobic oxidation induced by air sparging. The initial points correspond to $1 \mathrm{D}$ ${ }^{31} \mathrm{P}$ spectra, and the latter correspond to the sel-SHARPER spectra. The spectra, plotted on the same scale and acquired at the changeover point of the monitoring method, are shown together with their attributes. Full details are given in Figure S14.

analyzed in detail to provide guidelines for its most effective application to ${ }^{19} \mathrm{~F},{ }^{1} \mathrm{H}$, and ${ }^{31} \mathrm{P}$. SHARPER effects hetero- and homonuclear decoupling of all nuclei without the need to pulse on the $\mathrm{X}$ channel, yielding significant improvements in achievable $\mathrm{S} / \mathrm{N}$ ratio. It is also insensitive to magnetic field inhomogeneity, either static or, within certain limits, dynamic inhomogeneity, making it a valuable tool for quantitative monitoring of chemical reactions and parameters of chemical exchange in challenging environments. These attributes were exemplified by several examples, included protodeboronation of polyfluorinated aryl boronic acids, an investigation of a very fast accompanying boronic acid/boronate equilibrium, and oxidation of diphenylphosphine induced by intense air bubbling.

It is worth noting that, depending on the exchange parameters and within the constraints of the $J$ removal requirements of the SHARPER experiments, it may be possible explore the effects of varying CPMG frequency by changing the chunk time. In this way, much slower dynamics can be studied as demonstrated in relaxation dispersion experiments. ${ }^{28}$ The SHARPER methodology will also find applications outside of reaction monitoring, e.g., a simple modification of the pulse sequence offers a direct route toward accurate measurement of small homo- and heteronuclear scalar and dipolar coupling constants The SHARPER building block is expected to be applied in other branches of NMR, e.g., in vivo spectroscopy or $\mathrm{MRI}$, and thus to play a role beyond improving the $\mathrm{S} / \mathrm{N}$ ratio of high-resolution NMR spectra of high-abundance spins. SHARPER also provides a direct avenue for dealing with inherently poor magnetic field homogeneity of the upcoming benchtop NMR spectrometers, where the sensitivity gains are expected to be substantial.

\section{ASSOCIATED CONTENT}

\section{S Supporting Information}

The Supporting Information is available free of charge on the ACS Publications website at DOI: 10.1021/acs.analchem.7b02437.

SHARPER pulse sequences, ${ }^{19} \mathrm{~F}$ spectra and coupling constants, effects of magnetic field inhomogeneity, chunk length and miss setting of the resonance offset and pulses, reproducibility of integrals under bubbling conditions, ${ }^{1} \mathrm{H}$ SHARPER spectra of vincamine, reaction monitoring spectra, ${ }^{19} \mathrm{~F}$ linewidths of fluorinated boronic acids and interpretation of the boronic acid/boroanate dynamics, and ${ }^{31} \mathrm{P}$ spectra monitoring diphenylphosphine oxidation initiated by bubbling air (PDF)

\section{AUTHOR INFORMATION}

\section{Corresponding Authors}

*E-mail: guy.lloyd-jones@ed.ac.uk (G.C.L.-J.).

*E-mail: dusan.uhrin@ed.ac.uk (D.U.).

ORCID $\odot$

Guy C. Lloyd-Jones: 0000-0003-2128-6864

Dušan Uhrín: 0000-0002-0254-4971

\section{Notes}

The authors declare no competing financial interest.

\section{ACKNOWLEDGMENTS}

The research leading to these results received funding from the European Research Council under the European Union's Seventh Framework Programme (FP7/2007-2013)/ERC grant agreement no. [340163]. We thank Edward King ( $\mathrm{TgK}$ Scientific), Dr. Ruth Dooley (Edinburgh), and Dr. Anna Codina (Bruker) for their technical advice throughout this project. A.B. Jones is in part funded by Bruker UK.

\section{REFERENCES}

(1) Zangger, K. Prog. Nucl. Magn. Reson. Spectrosc. 2015, 86-87, 1.

(2) Nilsson, M.; Morris, G. A. Chem. Commun. 2007, 933.

(3) Castanar, L.; Parella, T. Magn. Reson. Chem. 2015, 53, 399.

(4) Aguilar, J. A.; Faulkner, S.; Nilsson, M.; Morris, G. A. Angew. Chem., Int. Ed. 2010, 49, 3901.

(5) Morris, G. A.; Aguilar, J. A.; Evans, R.; Haiber, S.; Nilsson, M. J. Am. Chem. Soc. 2010, 132, 12770.

(6) Lupulescu, A.; Olsen, G. L.; Frydman, L. J. Magn. Reson. 2012, 218,141 .

(7) Meyer, N. H.; Zangger, K. Angew. Chem., Int. Ed. 2013, 52, 7143.

(8) Paudel, L.; Adams, R. W.; Kiraly, P.; Aguilar, J. A.; Foroozandeh, M.; Cliff, M. J.; Nilsson, M.; Sandor, P.; Waltho, J. P.; Morris, G. A. Angew. Chem., Int. Ed. 2013, 52, 11616.

(9) Castanar, L.; Nolis, P.; Virgili, A.; Parella, T. Chem. - Eur. J. 2013, $19,17283$.

(10) Zangger, K.; Sterk, H. J. Magn. Reson. 1997, 124, 486.

(11) Bruschweiler, R.; Griesinger, C.; Sorensen, O. W.; Ernst, R. R. J. Magn. Reson. 1988, 78, 178.

(12) Krishnamurthy, V. V. Magn. Reson. Chem. 1997, 35, 9.

(13) Ying, J. F.; Roche, J. L.; Bax, A. J. Magn. Reson. 2014, 241, 97.

(14) Carr, H. Y.; Purcell, E. M. Phys. Rev. 1954, 94, 630.

(15) Meiboom, S.; Gill, D. Rev. Sci. Instrum. 1958, 29, 688.

(16) Freeman, R.; Hill, H. D. W. J. Chem. Phys. 1971, 54, 301.

(17) Gochin, M.; Weitekamp, D. P.; Pines, A. J. Magn. Reson. 1985, 63, 431.

(18) Stejskal, E. O.; Tanner, J. E. J. Chem. Phys. 1965, 42, 288.

(19) Cox, P. A.; Leach, A. G.; Campbell, A. D.; Lloyd-Jones, G. C. J. Am. Chem. Soc. 2016, 138, 9145.

(20) Ndukwe, I. E.; Shchukina, A.; Kazimierczuk, K.; Butts, C. P. Chem. Commun. 2016, 52, 12769.

(21) Bauer, C.; Freeman, R.; Frenkiel, T.; Keeler, J.; Shaka, A. J. J. Magn. Reson. 1984, 58, 442.

(22) Huang, Y.; Cao, S.; Yang, Y.; Cai, S.; Zhan, H.; Tan, C.; Lin, L.; Zhang, Z.; Chen, Z. Anal. Chem. 2017, 89, 7115.

(23) Wagner, G. E.; Sakhaii, P.; Bermel, W.; Zangger, K. Chem. Commun. 2013, 49, 3155.

(24) Ishihara, K.; Nagasawa, A.; Umemoto, K.; Ito, H.; Saito, K. Inorg. Chem. 1994, 33, 3811.

(25) Wennerstrom, H. Mol. Phys. 1972, 24, 69.

(26) Rogers, M. T.; Woodbrey, J. C. J. Phys. Chem. 1962, 66, 540. 
(27) Kono, Y.; Ishihara, K.; Nagasawa, A.; Umemoto, K.; Saito, K. Inorg. Chim. Acta 1997, 262, 91.

(28) Orekhov, V. Y.; Pervushin, K. V.; Arseniev, A. S. Eur. J. Biochem. 1994, 219, 887. 